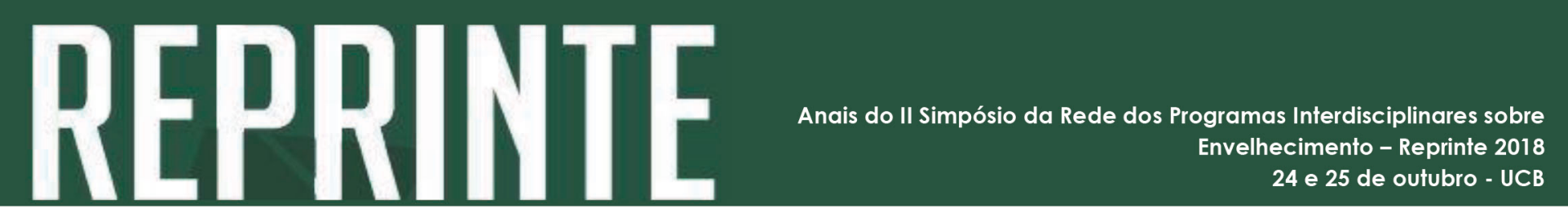

http://dx.doi.org/10.5335/rbceh.v16i1.9794

\title{
2) Interdisciplinaridade voltada ao envelhecimento humano
}

Dione Maria Setti Frizon ${ }^{1}$; Nadir Antonio Pichler²; Helenice de Moura Scortegagna²

\section{Resumo}

O atendimento eficaz aos idosos necessita ter enfoque na interdisciplinaridade. Assim, o objetivo do estudo é analisar a relevância e eficácia do atendimento multiprofissional ou interdisciplinar nos cuidados de saúde dos idosos. Acredita-se que o envelhecimento como um fenótipo complexo requer pesquisas integrando conceitos das humanidades, ciências sociais, epidemiologia, neurologia, fisiologia e biologia molecular. O tipo de pesquisa é bibliográfica sobre a Interdisciplinaridade dos profissionais voltada ao envelhecimento humano. Os resultados apontaram que, através de uma variedade de métodos, o atendimento interdisciplinar pode compartilhar um compromisso com a pesquisa baseada na comunidade que visa apoiar a colaboração, capacitar as comunidades e, finalmente, transformar práticas e políticas para melhor atender às diversas necessidades dos idosos urbanos e rurais.

Palavras-chave: Interdisciplinaridade. Saúde do Idoso. Atendimento Eficaz.

\section{0 atendimento interdisciplinar nos cuidados de saúde dos idosos}

A presente pesquisa tem o objetivo de analisar a relevância e eficácia do atendimento multiprofissional ou interdisciplinar nos cuidados de saúde dos idosos. Por isso, a medida que seus recursos pessoais, sociais e práticos diminuem, as pessoas idosas são capazes de compensar muitas perdas, por meio da participação social com amor e carinho de quem é próximo. Mas há uma fase crítica em que as pessoas perdem a possibilidade de compensação e consideram o lar como último recurso para manter seu bem-estar. Assim, para Veras (2009), o envelhecimento global requer acesso a recursos econômicos, apoio a saúde e o bem-estar, independente da diversidade cultural e geográfica, diante das crescentes desigualdades estruturais. Neste contexto, o uso de tecnologia para a avaliação do funcionamento físico em idosos para a prática clínica é primordial. Na visão de Araújo et al. (2011), existe relação das medidas de desempenho físico com o início da incapacidade, hospitalização, internação em

1 Mestranda em Ciência do Envelhecimento Humano. Endereço para correspondência: Universidade de Passo Fundo, BR 285, São José I Passo Fundo/RS I CEP: 99052-900. Email: dionesetti@hotmail.com

2 Professores no Programa de Pós-Graduação em Envelhecimento Humanod da Universidade de Passo Fundo (UPF). Emails (na ordem em que aparecem): nadirp@upf.br; helenice@upf.br 
lares de idosos, quedas, mortalidade e outros desfechos. Embora as medidas de desempenho estejam sendo usadas com mais frequência em pesquisas sobre envelhecimento, sua aceitação na prática clínica tem sido lenta, em parte devido à carga adicional no atendimento clínico de pacientes geriátricos.

O envelhecimento como um fenótipo complexo requer pesquisas integrando conceitos das humanidades, ciências sociais, epidemiologia, neurologia, fisiologia e biologia molecular. Segundo Veras (2009), o rápido crescimento da população idosa brasileira apresenta desafios únicos para os cuidados de saúde e os cuidados geriátricos podem ser complexos e demorados, e muitos médicos, psicossociais e questões funcionais devem ser abordadas simultaneamente. Para que o tratamento seja eficaz, deve ser cuidadosamente coordenado.

A área Interdisciplinar deve-se pautar, logicamente, com Programas stricto sensu que contenham linhas de pesquisas, projetos de pesquisas, grade curricular e objetivos que enalteçam a interdisciplinaridade. Sobre a estrutura dos Programas, o Documento da CAPES APCN (2017) destaca que, em linhas gerais, um programa de pós-graduação na Área Interdisciplinar deve conter proposta integradora, com poucas áreas de concentração, objetivos focalizados, linhas de pesquisa e projetos igualmente integradores, visando formar mestres e doutores com perfil inovador, assegurado por disciplinas ministradas por dois ou mais docentes com diferentes formações e de forma compartilhada.

De acordo com Santos et al. (2015), a falta de um entendimento claro sobre como a interdisciplinaridade pode ser colocada em prática, faz com que os professores, de modo geral, tenham dificuldades na construção de um ensino e pesquisa que seja capaz de associar conteúdo de diferentes disciplinas. Neste sentido Chena et al. (2015) explicam que o uso de uma equipe interdisciplinar é um método aceito e bem desenvolvido para coordenação do cuidado. A sua formação já está integrada nos currículos de alguns programas de formação profissional, incluindo programas em odontologia, medicina geriátrica, medicina paliativa, enfermagem, trabalho social, farmácia e psicologia.

Compreende-se que formar novas competências emerge no cerne do saber e da produção de conhecimento. Esse saber, segundo Motta e Aguiar (2007), extrapola a mera agregação dos seus campos de origem, visando à associação dialética entre dimensões polares como teoria e prática, ação e reflexão, conteúdo e processo. No entanto, a educação formal no trabalho permanece inadequada na maioria dos programas de treinamento profissional. Além de ser treinada em disciplinas com aspectos específicos dos cuidados geriátricos, todos os profissionais de saúde devem ser treinados para trabalhar numa equipe interdisciplinar em funcionamento, a fim de fornecer o melhor atendimento para os idosos. Ressalta-se a importância da criação e expansão de programas acadêmicos e de educação continuada em treinamento de equipe interdisciplinar, incluindo associações profissionais, órgãos de credenciamento e licenciamento, organizações de credenciamento e administradores universitários, bem como o uso da tecnologia para melhorar a simplicidade e a eficiência na prática clínica.

Globalmente, os idosos estão experimentando ganhos na expectativa de vida, resultando anos de vida saudáveis. Araújo et al. (2011) afirmam que fatores externos, como avanços nos tratamentos, contribuem para o envelhecimento saudável, mas evidências sugerem que fato- 
res internos ao indivíduo também são influentes. Uma delas, a religiosidade, é cada vez mais reconhecida como um potencial contribuinte para vidas mais longas e saudáveis.

\section{Material e Métodos}

De acordo com Minayo (2012), a metodologia é o caminho do pensamento e a prática exercida na abordagem da realidade e inclui, simultaneamente, a teoria de abordagem (o método), os instrumentos de operacionalização do conhecimento (as técnicas) e a criatividade do pesquisador, ou seja, sua experiência, sua capacidade pessoal e sua sensibilidade.

O tipo de pesquisa é bibliográfica, com abordagem qualitativa, visando subsidiar futuras pesquisas sobre a presente temática, identificando fatores relevantes sobre a interdisciplinaridade na área de saúde relacionada aos idosos.

Segundo Marconi e Lakatos (2003), a revisão de literatura é um apanhado geral sobre os principais trabalhos já realizados, revestidos de importância, por serem capazes de fornecer dados atuais e relevantes relacionados com o tema. O estudo de literatura pertinente poder ajudar a planificação do trabalho, evitar publicações e certos erros, e representa uma fonte indispensável de informações, podendo até orientar as indagações.

\section{Resultados/Conclusões}

Chena et al. (2015) relatam que a interdisciplinaridade dos profissionais desempenha um papel essencial na compreensão e abordagem das necessidades que emergem de disparidades e interseccionalidades que moldam a vida dos idosos em comunidades multiculturais.

Através de uma variedade de métodos, o atendimento interdisciplinar pode compartilhar um compromisso com a pesquisa baseada na comunidade que visa apoiar a colaboração, capacitar as comunidades e, finalmente, transformar práticas e políticas para melhor atender às diversas necessidades dos idosos urbanos e rurais. Araújo et al (2011) ressaltam, ainda, que o envelhecimento saudável deveria ser base para algum programa de melhoria de saúde promovido pelo Governo Federal, possuindo centros regionais de educação e clínicas de saúde em todo o país para melhorar o acesso à assistência geriátrica, aumentar a alfabetização em saúde, promover estilos de vida saudáveis e práticas preventivas, assim como treinar cuidadores familiares e profissionais.

Vale ressaltar que melhorar a política de saúde e envelhecimento, particularmente com foco em ambientes rurais e populações indígenas, é um grande desafio, pois, esses componentes incluiriam um programa de treinamento do cuidador sofisticado, rede de telemedicina dos hospitais rurais, e várias atividades de educação superior e promoção da saúde baseadas em evidências. Os resultados dessa prática são positivos, sendo possível compreender de forma expandida do envelhecimento humano, os campos de trabalho e a necessidade de desempenhos interdisciplinares em futuras intervenções, abordando as principais teorias e aspectos da subjetividade em idade.

Desta forma, Guimarães (2012) considera que demandas decorrentes do envelhecimento populacional exigem profissionais competentes em suas áreas de formação, mas também que 
possuam atitude interdisciplinar. Independentemente do ambiente de cuidados, os complexos problemas de saúde dos adultos mais velhos, necessitam de múltiplos profissionais de saúde e a coordenação do cuidado entre toda a equipe de profissionais, trabalhadores de assistência direta e familiares e outros cuidadores.

Santos et al. (2011) acreditam que quando os cuidados de saúde são abordados unilateralmente, o cuidado pode ser tornar fragmentado e não nas necessidades gerais da pessoa. Por exemplo, vários problemas de saúde de um indivíduo podem ser propriamente diagnosticados, com tratamentos apropriados escolhidos, mas o indivíduo também pode ter problemas psicológicos que impedem sua compreensão desses tratamentos. Chena et al. (2015) explicam que no atendimento interdisciplinar, todas essas necessidades podem ser abordadas de forma proativa e simultânea, com os fornecedores realizando metas comuns e produzindo um plano de cuidado abrangente e bem concebido.

De acordo com evidências da pesquisa de Guimarães (2012), o uso de uma equipe interdisciplinar no cuidado de idosos pode levar a uma melhor continuidade e qualidade dos cuidados, com melhores resultados de saúde e menores custos. Outros benefícios do atendimento em equipe incluem maior comunicação entre os prestadores de serviços de saúde, melhoria da segurança do paciente, melhor atendimento de doenças crônicas comuns, melhor medicação e aderência, menos reações adversas a medicamentos, preservação da função e diminuição das readmissões. $\mathrm{O}$ cuidado interdisciplinar também tem sido demonstrado ser útil no ambulatório, bem como em instalações de enfermagem especializadas.

Para Santos et al. (2011), os programas de treinamento em cuidado interdisciplinar podem melhorar o conhecimento e as atitudes dos alunos em relação ao envelhecimento, cuidados, habilidades de equipe, comunicação interprofissional e benefícios da colaboração.

\title{
Interdisciplinarity focused on human aging
}

\begin{abstract}
Effective care for the elderly needs to focus on interdisciplinarity. Thus, the objective of the study is to analyze the relevance and effectiveness of multiprofessional or interdisciplinary care in the health care of the elderly. It is believed that aging as a complex phenotype requires research integrating concepts from the humanities, social sciences, epidemiology, neurology, physiology and molecular biology. The type of research is a bibliographical one on the Interdisciplinarity of the professionals directed to the human aging. The results pointed out that through a variety of methods, interdisciplinary care can share a commitment to community-based research that aims to support collaboration, empower communities and ultimately transform practices and policies to better address the diverse needs of older people urban and rural areas.
\end{abstract}

Keywords: Interdisciplinarity. Health of the Elderly. Effective Service. 


\section{Referências}

ARAÚJO, L.F. et al. Evidências da contribuição dos programas de assistência ao idoso na promoção do envelhecimento saudável no Brasil. Rev. Panam Salud Publica, (impr.), v. 30, n.1, p. 80-86, 2011.

BRASIL. CAPES APCN. Requisitos para a Apresentação de Propostas de Cursos Novos (APCN). Brasília: 2017.

CHENA, D.N.C. et al. Envelhecimento e interdisciplinaridade: análise da produção científica da revista estudos interdisciplinares sobre o envelhecimento. Estud. interdiscipl. envelhec., (impr.), Porto Alegre, v. 20, n. 3, p. 883-901, 2015.

GUIMARÃES, A.C.A et al. Percepção da qualidade de vida e da finitude de adultos de meia idade e idoso praticantes e não praticantes de atividade física. Rev. bras. geriatr. gerontol. (impr.), Rio de Janeiro, v.15, n. 4, p. 23-34, oct./dec. 2012.

MARCONI M.A; LAKATOS E.M. Fundamentos de metodologia científica. 5. ed. - São Paulo: Atlas, 2003.

MINAYO, M.C.S. Pesquisa social: teoria, método e criatividade. 32. ed. Petrópolis: Editora Vozes, 2012.

MOTTA, L.B; AGUIAR, A.C. Novas competências profissionais em saúde e o envelhecimento populacional brasileiro: integralidade, interdisciplinaridade e intersetorialidade. Ciência \& Saúde Coletiva, Rio de Janeiro, v.12, n.2, p. 363-372, mar/abr. 2007.

SANTOS, I. et al. O grupo pesquisador construindo ações de autocuidado para o envelhecimento saudável: pesquisa sociopoética. Esc. Anna Nery (impr.), v.15, n. 4, p.746-754, out/dez, 2011.

VERAS, R. Envelhecimento populacional contemporâneo: demandas, desafios e inovações. Revista de Saúde Pública, São Paulo, v. 43, n. 3, p. 548-554, maio/jun. 2009. 